\title{
ÉSTERES TRITERPÊNICOS DE HIMATANTHUS SUCUUBA (SPRUCE) WOODSON
}

\section{Jefferson Rocha de A. Silva, Claudia M. Rezende e Ângelo C. Pinto}

Instituto de Química - Universidade Federal do Rio de Janeiro - Cidade Universitária - Ilha do Fundão - C. T.; Bloco A - 21945-970 - Rio de Janeiro - RJ

Maria L. B. Pinheiro, Milade C. Cordeiro e Everaldo Tamborini

Departamento de Química - Universidade Federal do Amazonas - Campus Universitário - Mini-Campus - Estrada do Contorno no 3000 - Japiim - 69077-000 - Manaus - AM

Cláudia M. Young

Instituto de Botânica de São Paulo - CP 4005 - 01061-970 - São Paulo - SP

Vanderlan da S. Bolzani

Instituto de Química - Universidade Estadual Paulista - 14800-900 - Araraquara - SP

Recebido em 17/7/97; aceito em 14/5/98

\begin{abstract}
TRITERPENIC ESTERS FROM HIMATANTHUS SUCUUBA (SPRUCE) WOODSON. Bioactivityguided fractionation from hexane extract of Himatanthus sucuuba barks utilizing Cladosporium sphaerospermum led to the isolation of iridoids plumericin and isoplumericin, which showed higher inhibition against $C$. sphaerospermum than the antibiotic nistatin. Besides bioactive iridoids were isolated the inactive triterpenes lupeol cinnamate, $\alpha$-amyrin cinnamate and lupeol acetate.
\end{abstract}

Keywords: Himatanthus sucuuba; iridoids; triterpenes.

\section{INTRODUÇÃO}

Himatanthus sucuuba (Spruce) Woodson (Apocynaceae) é uma planta conhecida na região norte do Brasil como sucuuba, janaguba ou sucuba. Esta planta é amplamente utilizada na medicina popular como antitumoral, antifúngica, vermífuga e anti-anêmica ${ }^{1}$.

Estudos anteriores revelaram a presença de depsídeos, terpenos e iridóides em H.sucuuba ${ }^{2,3}$. Dentre os iridóides foram encontrados a fulvoplumierina, isoplumericina e plumericina, de comprovada ação antineoplásica, antiflogística e antimicrobiana ${ }^{4-10}$. Os depsídeos isolados de H.sucuuba apresentaram atividade como inibidores da enzima beta monoamino-oxidase (MAO-B) ${ }^{3}$. No entanto, como foram encontrados nas cascas podem não ser provenientes da planta e sim de líquens infestantes.

No presente trabalho são descritos os resultados do estudo fitoquímico do extrato hexânico das cascas do caule de H.sucuuba. Este extrato é constituído basicamente por uma mistura de ésteres triterpênicos, que corresponde aproximadamente a 7\% do peso do extrato. Estes ésteres triterpênicos já foram descritos no gênero Himatanthus ${ }^{11,12}$. A atividade fungicida apresentada pelo extrato foi monitorada por bioautografia, utilizando-se o fungo fitopatogênico Cladosporium sphaerospermum ${ }^{13-14}$. A análise das frações bioativas por CGAREM, RMN ${ }^{1} \mathrm{H}$ e ${ }^{13} \mathrm{C}$ revelou a presença dos iridóides plumericina 1 e isoplumericina 2.

\section{RESULTADOS E DISCUSSÃO}

O extrato hexânico das cascas do caule foi submetido a bioautografia, utilizando o fungo Cladosporium sphaerospermum. A cromatoplaca de sílica foi eluída com uma mistura de hexano e acetato de etila (6:4). Uma zona de inibição intensa na cromatoplaca indicou a presença de substâncias fungitóxicas.

O extrato hexânico foi fracionado em coluna de SEPHADEXLH 20, usando hexano, diclorometano, acetona e metanol como eluentes. Das oito frações obtidas, a segunda e a terceira frações apresentaram zona de inibição na bioautografia. A cromatografia gasosa acoplada a espectrometria de massas destas duas frações mostrou a presença dos iridóides plumericina $\mathbf{1}$, isoplumericina
2, e de uma mistura de ésteres triterpênicos das séries lupano e ursano, como constituintes em maior proporção na mistura.

Ao extrato hexânico dissolvido em clorofórmio adicionouse etanol. Este procedimento levou à obtenção de um sólido amorfo que foi cromatografado em coluna de gel de sílica. A eluição da coluna com uma mistura de hexano/benzeno em gradiente de polaridade crescente forneceu uma mistura de duas substâncias e um sólido cristalino. A análise da mistura, por cromatografia gasosa acoplada a espectrometria massas, mostrou o mesmo íon molecular de m/z 556 para as duas substâncias, compatível com a fórmula molecular $\mathrm{C}_{39} \mathrm{H}_{56} \mathrm{O}_{2}$. Foram observados íons característicos de triterpenos das séries lupano de $\mathrm{m} / \mathrm{z} 189(32 \%)$ e $\Delta^{12}$-urseno ou oleaneno de $\mathrm{m} / \mathrm{z} 218(46 \%)$, este último proveniente de um rearranjo de tipo retro- DielsAlder $^{15-16}$. O íon de $\mathrm{m} / \mathrm{z} 408\left(\mathrm{M}^{+\bullet}-148\right)$, resultante da perda de $\mathrm{C}_{9} \mathrm{H}_{8} \mathrm{O}_{2}$, acompanhado do fragmento de m/z 131 (100\%) levaram a suposição da presença de um grupamento cinamoíla no esqueleto triterpênico ${ }^{17-18}$

Os espectros de RMN ${ }^{13} \mathrm{C}$ (desacoplado e DEPT $90^{\circ}$ e $135^{\circ}$ ) da mistura registraram os pares de deslocamentos químicos 124.5 $(\mathrm{CH})$ e $139.8(\mathrm{C})$, e $109.6\left(\mathrm{CH}_{2}\right)$ e $151,1(\mathrm{C})$, característicos para as ligações duplas $C(12)-C(13)$ da série urseno e $C(20)$ $\mathrm{C}(29)$ do grupo isopropilideno do esqueleto lupano ${ }^{19-20}$, respectivamente. A unidade cinamoila foi confirmada através dos deslocamentos químicos em $\delta$ 128,2 (C-2' e C-6'), 129,0 (C-3'e C5'), 130,3 (C-4') e 134,3 (C-1') do anel aromático e em 119,0, 144,4 e 166,3 da unidade $(\mathrm{CH}=\mathrm{CH}-\mathrm{COO})$. O par de dubletos no $\mathrm{RMN}{ }^{1} \mathrm{H}$ em $\delta 7,70(1 \mathrm{H}, \mathrm{J}=16 \mathrm{~Hz})$ e $6,48(1 \mathrm{H}, \mathrm{J}=16 \mathrm{~Hz})$ e 7,65 $(1 \mathrm{H}, \mathrm{J}=16 \mathrm{~Hz})$; e $6,42(1 \mathrm{H}, \mathrm{J}=16 \mathrm{~Hz}$.$) mostram que a ligação$ dupla tem configuração $\mathrm{E}^{21-22}$. A hidrólise básica desta mistura levou a obtenção de ácido cinâmico, lupeol e $\alpha$-amirina ${ }^{23}$, cujas as estruturas foram confirmadas através de seus espectros de massas (CGAR-EM) e co-injeção com padrões.

O sólido cristalino 5 mostrou o íon molecular de $\mathrm{m} / \mathrm{z} 468$, compatível com a fórmula molecular $\mathrm{C}_{32} \mathrm{H}_{52} \mathrm{O}_{2}$, e fragmentos no espectro de massas característicos do esqueleto lupano ${ }^{19-21,24-26}$.

A unidade acetil foi caracterizada pelos espectros de RMN ${ }^{1} \mathrm{H}$ e ${ }^{13} \mathrm{C}$ de $\mathbf{5}^{19-21,24-26}$, que foi identificado como acetato de lupeol.

$\mathrm{O}$ fracionamento guiado por bioensaio das frações bioativas levou à obtenção de uma mistura dos iridóides $\mathbf{1}$ e $\mathbf{2}$. A análise 
por CGAR-EM mostrou que ambos os iridóides tem o mesmo peso molecular $\left(\mathrm{M}^{+\bullet} 290, \mathrm{C}_{15} \mathrm{H}_{14} \mathrm{O}_{6}\right)$. Os fragmentos de $\mathrm{m} / \mathrm{z} 258$ $\left(\mathrm{M}^{+\bullet}-32,42 \%\right)$ e de $\mathrm{m} / \mathrm{z} 230\left(\mathrm{M}^{+\bullet}-60,85 \%\right)$ são provenientes da perda de $\mathrm{MeOH}$ e de formiato de metila, enquanto o de m/z 193 $\left(\mathrm{M}^{+\bullet}-97,78 \%\right)$ é resultante da perda do anel da lactona.

$\mathrm{O}$ espectro de $\mathrm{RMN}{ }^{1} \mathrm{H}$ desta mistura registrou um par de dubletos de quartetos em $\delta 7,14(1 \mathrm{H}, \mathrm{J}=1,0$ e 7,0 Hz) e $6,80(1 \mathrm{H}$, $\mathrm{J}=1,0$ e 7,0 Hz), que foram atribuídos ao hidrogênio olefínico no C-13 da plumericina $\mathbf{1}$ e isoplumericina $\mathbf{2}$, respectivamente.

A avaliação biológica foi feita utilizando a suspensão de esporos do fungo fitopatogênico Cladosporium sphaerospermum. Pelo método da autobiografia utilizando diferentes concentrações das substâncias $\mathbf{1}$ e $\mathbf{2}$, verificou-se que o limite de deteção, tanto para plumericina $\mathbf{1}$ quanto para isoplumericina $\mathbf{2}$ está abaixo de $1 \mu \mathrm{g}$. Este resultado indica uma atividade fungitóxica para estes iridóides cinco vezes maior do que o observado para o antibiótico nistatina $(5 \mu \mathrm{g})$, usado como padrão.
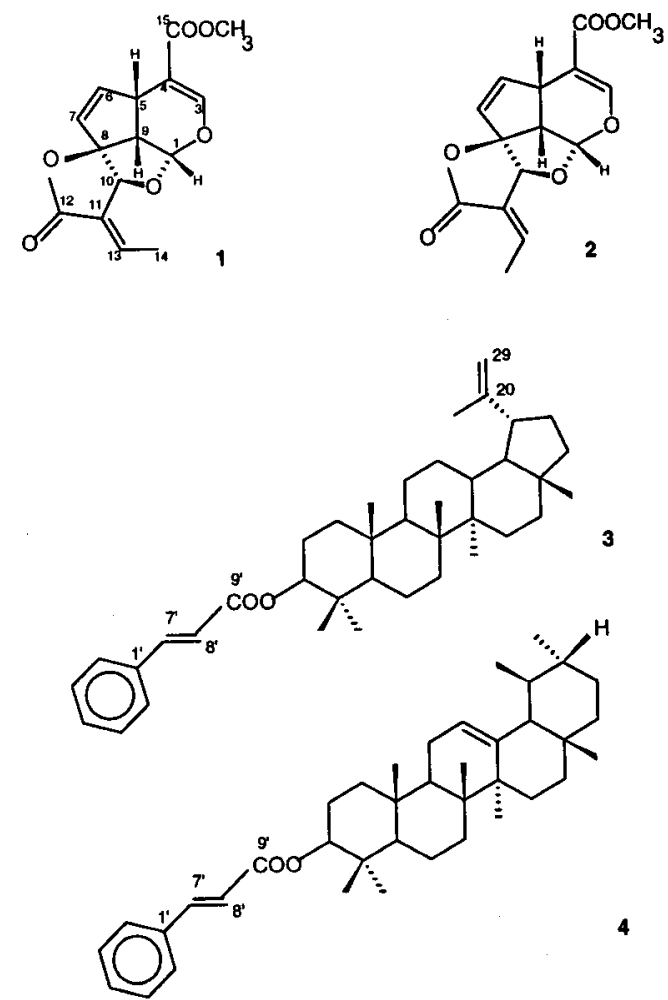<smiles>C=C(C)[C@@H]1CCC2(C)CC[C@]3(C)C(CCC4C(CC[C@@]5(C)C(C)(C)C(OC)CCC45C)C3(C)C)C12</smiles>

\section{EXPERIMENTAL}

Planta - Coletada em janeiro de 1995 no município de Santarém, estado do Pará. A exsicata está depositada no Herbário do Instituto de Ciências Biológicas da Universidade do Amazonas, Manaus, Amazonas, Brasil, registrada sob № 5436 .

Extração e separação - $350 \mathrm{~g}$ de material botânico (casca do caule) de Himatanthus sucuuba, seco e moído, foram submetidos a extração em aparelho Soxhlet por 3 dias, utilizando-se sucessivamente, hexano, clorofórmio e etanol. O resíduo hexânico $(28,7 \mathrm{~g})$, de coloração laranja e consistência pastosa, foi tratado com clorofórmio e etanol, precipitando $2,0 \mathrm{~g}$ de um sólido branco amorfo. $200 \mathrm{mg}$ deste sólido foram cromatografados em coluna de gel de sílica (hexano/benzeno em gradiente crescente de polaridade), fornecendo uma mistura constituída de 3 e $4(58,8 \mathrm{mg})$ e um sólido $5(25,2 \mathrm{mg})$. $6,0 \mathrm{~g}$ da fração hexânica bruta foram ainda cromatografados em coluna aberta utilizando SEPHADEX LH-20 (60 g) como fase estacionária e como eluentes hexano, diclorometano, acetona e metanol em gradiente de polaridade crescente fornecendo: (A) $\mathrm{C}_{6} \mathrm{H}_{14}(5,0 \mathrm{~g})$; (B) $\mathrm{C}_{6} \mathrm{H}_{14}: \mathrm{CH}_{2} \mathrm{Cl}_{2}(8: 2 ; \mathrm{m}=0,5 \mathrm{~g}) ;(\mathbf{C}) \mathrm{C}_{6} \mathrm{H}_{14}: \mathrm{CH}_{2} \mathrm{Cl}_{2}(6: 4 ; \mathrm{m}=0,3$ g); (D) $\mathrm{C}_{6} \mathrm{H}_{14}: \mathrm{CH}_{2} \mathrm{Cl}_{2}(2: 8 ; \mathrm{m}=0,05 \mathrm{~g}) ;$ (E) $\mathrm{CH}_{2} \mathrm{Cl}_{2}: \mathrm{C}_{3} \mathrm{H}_{6} \mathrm{O}$ (8:2; $\mathrm{m}=0,08 \mathrm{~g}) ;(\mathbf{F}) \mathrm{CH}_{2} \mathrm{Cl}_{2}: \mathrm{C}_{3} \mathrm{H}_{6} \mathrm{O}(6: 4 ; \mathrm{m}=0,03 \mathrm{~g}) ;(\mathbf{G}) \mathrm{CH}_{2} \mathrm{Cl}_{2}: \mathrm{C}_{3} \mathrm{H}_{6} \mathrm{O}$ $(2: 8 ; \mathrm{m}=0,008 \mathrm{~g}) ;(\mathbf{H}) \mathrm{MeOH}(\mathrm{m}=0,002 \mathrm{~g})$.

A cromatografia em camada fina das frações obtidas indicou a presença de substâncias com o mesmo Rf nas frações B e C. Estas frações foram reunidas $(\mathrm{m}=0,8 \mathrm{~g})$ e cromatografadas em coluna de gel de sílica tendo como eluentes hexano, diclorometano, acetato de etila e metanol em gradiente de eluição. Após a bioautografia das frações (vide procedimento abaixo), a fração eluída com $\mathrm{CH}_{2} \mathrm{Cl}_{2}$ : AcOEt $(8: 2,108 \mathrm{mg})$ foi cromatografada em coluna de gel de sílica com hexano/acetato de etila em gradiente de eluição, obtendo-se oito frações das quais a fração eluída com $\mathrm{C}_{6} \mathrm{H}_{14}$ : AcOEt (7:3) é uma mistura constituída pelos iridóides 1 e 2 (16,3 mg).

Hidrólise da mistura de 3 e 4 - $45 \mathrm{mg}$ deste material foram refluxados com $6 \mathrm{ml}$ de solução metanólica de $\mathrm{KOH}$ a $5 \%$ em $60 \mathrm{ml}$ de hexano. Após evaporação do metanol e adição de água, extraiu-se a solução com clorofórmio, obtendo-se uma fração insaponificável, que foi identificada por co-injeção em CGAR como lupeol e $\alpha$-amirina. A fração aquosa alcalina acidificada com $\mathrm{HCl}$ e extraída com acetato de etila forneceu um sólido cristalino que foi identificado por comparação com amostra autêntica, através de CCD, IV e co-injeção em CGAR, como o ácido cinâmico.

Bioensaio - A análise do extrato hexânico foi realizada em cromatografia de camada fina, tendo como sistema de solventes hexano:acetato de etila (6:4).Após o registro das bandas cromatográficas que absorveram na luz ultravioleta, fez-se a aplicação da suspensão, contendo Cladosporium sphaerospermum, sobre a cromatoplaca com subsequente deposição em atmosfera úmida para incubação por um período de dois a três dias.

Suspensão de Cladosporium sphaerospermum - Uma solução contendo $\mathrm{KH}_{2} \mathrm{PO}_{4}(7 \mathrm{~g}), \mathrm{Na}_{2} \mathrm{HPO}_{4}$. $2 \mathrm{H}_{2} \mathrm{O}(3 \mathrm{~g}), \mathrm{KNO}_{3}(4$ g), $\mathrm{MgSO}_{4} \cdot 7 \mathrm{H}_{2} \mathrm{O}(1 \mathrm{~g})$ foi autoclavada a $120^{\circ} \mathrm{C}$ por $20 \mathrm{minu}-$ tos. A $60 \mathrm{ml}$ desta solução foram adicionados $10 \mathrm{ml}$ de uma solução de glicose a $30 \%$ e, em seguida, adicionou-se o fungo Cladosporium sphaerospermum até obter-se uma suspensão.

Dados físicos e espectrométricos: Plumericina (1). IV(KBr) $v_{\max } \mathrm{cm}^{-1}$ (mistura de 1 e 2): $1757,1707,1685$ e $1647 . \mathrm{EM} \mathrm{m} / \mathrm{z}$ (int.rel.): 290(30), 230(60), 201 (70), 193(80), 173 (40), 160 (70), $139(100)$ e $115(60)$. RMN ${ }^{1} \mathrm{H}\left(300 \mathrm{MHz}, \mathrm{CDCl}_{3}\right.$.): $\delta 7,14$ (dq, J=1,0 e 7,0 Hz, H-13), 6,04 (dd, J=2,0 e 5,0 Hz, H-6), 5,64 (dd, J=2,0 e 5,0 Hz, H-7), 5,30 (sl, H-10), 4,00 (m, H-5), 3,76 (COOMe), 2,01 (d, J=7,0 Hz, H-14). RMN ${ }^{13} \mathrm{C}(75,25$ $\left.\mathrm{MHz}, \mathrm{CDCl}_{3}\right)$ : 102,3 (C-1), 153 (C-3), 38,4 (C-5), 141,1 (C-6), 126,4 (C-7), 53,9 (C-9), 80,3 (C-10), 144,6 (C-13) ${ }^{27-29}$.

Isoplumericina (2). IV (KBr) $v_{\max } \mathrm{cm}^{-1}$ (mistura de 1 e 2): 1757, 1707,1685 e 1647. EM m/z (int.rel.): 290 (29), 230 (94), 201 (100), 193 (80), 173 (40), 160 (98), 139 (90) e 115 (60). RMN ${ }^{1}$ H.(300 MHz, $\mathrm{CDCl}_{3}$ ) : $\delta$ 6,80 (dq, J=1,0 e 7,0 Hz, H13 ), 6,04 (dd, J=2,0 e 5,0 Hz, H-6), 5,64 (dd, J=2,0 e 5,0 Hz, H-7), 5,30 (sl, H-10), 4,00 (m, H-5), 3,76 (COOMe), 2,01 (d, $\mathrm{J}=7,0 \mathrm{~Hz}, \mathrm{H}-14)$. RMN ${ }^{13} \mathrm{C}\left(75,25 \mathrm{MHz}, \mathrm{CDCl}_{3}\right): 102,3$ (C-1), 153 (C-3), 38,4 (C-5), 141,16 (C-6), 126,4 (C-7), 53,9 (C-9), $80,3$ (C-10), 144,6 (C-13) $)^{27-29}$.

Cinamato de lupeol (3). IV (KBr) $v_{\max } \mathrm{cm}^{-1}: 2940,2860$, 1709, 1638, 1458, 1171, 766 e $706 \mathrm{~cm}^{-1}$. EM m/z (int.rel.): 556[M] ${ }^{+}$(2), 408 (2), 337 (2), 218 (46), 190 (14), 189 (32), 131 
(100) e 103 (26). RMN ${ }^{1} \mathrm{H}\left(300 \mathrm{MHz}, \mathrm{CDCl}_{3}\right): \delta 7.70(\mathrm{~d}, \mathrm{~J}=16,0$ Hz, H-7'), 7,35-7,55 (m, Ar-H), 6,42 (d, J=16 Hz, H-8'), 4,67 (m, H-29b), 4,57 (m, H-29a). RMN ${ }^{13} \mathrm{C}$ (Tabela 1).

Cinamato de $\alpha$-amirina (4). $\mathrm{RMN}{ }^{1} \mathrm{H}\left(300 \mathrm{MHz}, \mathrm{CDCl}_{3}\right)$ : $\delta$ 7,65 (d, J=16,0 Hz, H-7), 7,35-7,55 (m, Ar-H), 6,48 (d, J=16,0 $\mathrm{Hz}, \mathrm{H}-8$ ) , 5,14 (t, J=3,4 Hz, H-12). RMN ${ }^{13} \mathrm{C}$ (Tabela 1).

Acetato de lupeol (5). p.f. $154-156^{\circ} \mathrm{C}$; IV $(\mathrm{KBr}) v_{\max } \mathrm{cm}^{-1}$ : 2950, 1734, 1244 e $1173 \mathrm{~cm}^{1}$. EM m/z: $468[\mathrm{M}]^{+}(11), 408$ (4), 218 (44), 189 (100), 121 (66). RMN ${ }^{1} \mathrm{H}\left(300 \mathrm{MHz}, \mathrm{CDCl}_{3}\right): \delta$ 4,69 (m), 4,57 (m), 2,04 (s), 1,03 (s), 0,94 (s), 0.85 (s) e 0.79 (s). RMN ${ }^{13} \mathrm{C}$ (Tabela 1)

Instrumental - Os espectros de $\mathrm{RMN}$ de ${ }^{1} \mathrm{H}$ e ${ }^{13} \mathrm{C}$ (300 e $75,25 \mathrm{MHz}$, respectivamente) foram obtidos em um espectrômetro Bruker, utilizando $\mathrm{CDCl}_{3}$ como solvente e TMS como padrão interno. A análise por CGAR (Cromatografia Gasosa de Alta Resolução) foi realizada em cromatógrafo HP5890 Condições: injetor: $260^{\circ} \mathrm{C}$; detetor por ionização de chama: $330^{\circ} \mathrm{C}$ (DIC), gás carreador $\mathrm{H}_{2}$; vazão: $2 \mathrm{ml} / \mathrm{min}$; temperatura programada: $250-320^{\circ} \mathrm{C}\left(5^{\circ} \mathrm{C} / \mathrm{min}\right), 320^{\circ} \mathrm{C}$ (30min); injeção com divisão de fluxo (20:1); coluna capilar de sílica fundida, fase estacionária $\mathrm{SE}-54,25 \mathrm{~m}, \mathrm{~d}_{\mathrm{ext}}=0,3 \mathrm{~mm}, \mathrm{~d}_{\text {fase }}=0,2 \mathrm{~nm}$. CGAR/EM - Cromatógrafo a gás HP-5880 acoplado a um espectrômetro de massas computadorizado HP-5897A com analisador de íons quadrupolo e ionização por impacto de elétrons, $70 \mathrm{eV}$; Temperatura programada: $40^{\circ}-320^{\circ} \mathrm{C}\left(10^{\circ} \mathrm{C} / \mathrm{min}\right)$; injeção on colunm, coluna capilar de sílica fundida, fase estacionária Silarem-30, 10m. $\mathrm{d}_{\mathrm{ext}}$ 0,3 mm. Os espectros de IV foram realizados em espectrômetro Nicolet: Magna-IR 760

Os fracionamentos cromatográficos foram realizados em coluna de vidro, empacotadas com Si 60-MERCK e SEPHADEX LH-20 (PHARMACIA); a CCD foi realizada em cromatofolhas MERCK PL, Si $60 \quad \mathrm{~F}_{254}, 0,2 \mathrm{~mm}$ de espessura. Os pontos de fusão foram obtidos em aparelho MICROQUÍMICA MQAPF301 e não foram corrigidos.

\section{AGRADECIMENTOS}

Os autores agradecem ao CNPq e ao PRONEX/FINEP $\mathrm{n}^{\circ}$ 41.96.00911.00 reg. 4002-96 pelo suporte financeiro.

\section{REFERÊNCIAS}

1. Van den Berg, M. E. Plantas Medicinais na Amazônia Contribuição ao seu conhecimento sistemático; CNPq/ PRU/MPEG; Belém, Brasil 1982.

2. Persinos, G. P.; Blomster, R.N.; J. Pharm. Sci. 1978, 67, 1322.

3. Endo, Y.; Hayashi, H.; Sato, T.; Maruno, M.; Ohta, T.; Nozoe, S.; Chem. Pharm. Bull. 1994, 42, 1198

4. Ferrigni, V. N. R.; Hasegawa, M.; Rev. Latinoamer. Quím. 1976, 7, 146.

5. Vanderlei, M. F.; Silva, M. S.; Gottlieb, H. E.; Braz-Filho, R.; J. Braz. Chem. Soc. 1991, 2, 51.

6. Kardono, L. B. S.; Tsauri, S.; Padmawinata, K.; Pezzuto, J. M.; Kinghorn, A. D.; J. Nat. Prod. 1990, 53, 1447.

7. Sanduja, R.; Weinheimer, A. J.; Euler, K. L.; Alam, M.; J. Nat. Prod. 1985, 48, 335.

8. Kupchan, S. M.; Dessertine, A. L.; Blaylock, B. T.; Bryan, R. F.; J. Org. Chem. 1974, 39, 2477.

9. Little, J. E.; Johnstone, D. B.; Arch. Biochem. 1951, 30, 445. 10. Anderson, J. E.; Chang, C. J.; Mclaughlin, J. L.; J. Nat. Prod. 1988, 51, 307.

11. Tan, G. T.; Pezzuto, J. M.; .Kinghorn, A. D .; Hughes, S. H.; J. Nat. Prod. 1991, 54, 143.

12. Coppen, J. J. W.; Phytochemistry 1983, 22, 179.

13. Homans, A. L.; Fuchs , A.; J. Chromatog. 1970, 51, 327.

14. Rahalison, L.; Hamburger, M.; Monod, M.; Frenk, E.; Hostettmann, K.; Planta. Med. 1994, 60, 41.

15. Budzikiewics, H.; Wilson, J. M.; Djerassi, C.; J. Am. Chem. Soc. 1963, 85, 3688.
Tabela 1. Dados espectrais (ppm) de $\mathrm{RMN}{ }^{13} \mathrm{C}$ de cinamato de lupeol (3), cinamato de $\alpha$-amirina (4) e acetato de lupeol (5).

\begin{tabular}{|c|c|c|c|}
\hline Carbono & 3 & 4 & 5 \\
\hline 1 & 38,6 & 38,6 & 38,6 \\
\hline 2 & 23,6 & 23,6 & 23,9 \\
\hline 3 & 81,2 & 81,2 & 81,2 \\
\hline 4 & 37,3 & 38,1 & 38,0 \\
\hline 5 & 55,5 & 55,5 & 55,5 \\
\hline 6 & 17,7 & 18,4 & 18,2 \\
\hline 7 & 34,4 & 32,8 & 34,4 \\
\hline 8 & 41,0 & 40,2 & 41,0 \\
\hline 9 & 50,5 & 47,8 & 50,5 \\
\hline 10 & 37,0 & 37,0 & 37,2 \\
\hline 11 & 21,1 & 17,7 & 21,1 \\
\hline 12 & 26,8 & 124,5 & 25,2 \\
\hline 13 & 38,1 & 139,8 & 38,2 \\
\hline 14 & 43,0 & 42,3 & 43,0 \\
\hline 15 & 27,6 & 28,6 & 27,6 \\
\hline 16 & 35,7 & 26,8 & 35,7 \\
\hline 17 & 43,0 & 33,9 & 43,0 \\
\hline 18 & 48,2 & 59,2 & 48,4 \\
\hline 19 & 47,8 & 39,8 & 48,2 \\
\hline 20 & 151,1 & 39,8 & 151,1 \\
\hline 21 & 29,9 & 31,3 & 29,9 \\
\hline 22 & 39,8 & 41,7 & 40,2 \\
\hline 23 & 28,2 & 28,2 & 28,1 \\
\hline 24 & 15,9 & 16,8 & 16,7 \\
\hline 25 & 16,1 & 15,9 & 16,2 \\
\hline 26 & 16,4 & 16,8 & 16,8 \\
\hline 27 & 14,7 & 23,4 & 14,7 \\
\hline 28 & 17,7 & 28,1 & 18,2 \\
\hline 29 & 109,6 & 23,4 & 109,5 \\
\hline 30 & 21,6 & 21,6 & 19,4 \\
\hline $1^{\prime}$ & 134,7 & 134,7 & 171,2 \\
\hline 2 , & 128,2 & 128,2 & 21,1 \\
\hline 3 , & 129,0 & 129,0 & - \\
\hline 4 , & 130,3 & 130,3 & - \\
\hline 5 , & 129,0 & 129,0 & - \\
\hline 6 & 128,2 & 128,2 & - \\
\hline 7 ' & 144,4 & 144,4 & - \\
\hline 8 & 119,0 & 119,0 & - \\
\hline 9', & 167,0 & 167,0 & - \\
\hline
\end{tabular}

16. Ogunkoya, L.; Phytochemistry 1981, 20, 121.

17. Delgado, M. C. C.; Silva, M. S.; Braz Filho, R.; Quim. Nova 1986, 9, 119.

18. Misra, G.; Mitra, C. R.; Phytochemistry 1968, 7, 2173.

19. Mahato, S. B.; Kundu, A. P.; Phytochemistry 1994, 37, 1517.

20. Olea, R. S. G.; Roque, N. F.; Quim. Nova 1990, 13, 278.

21. Bhattacharyya, J.; Barros, C. B.; Phytochemistry 1986, 25, 274.

22. Pan, H.; Lundgren, L. N.; Andersson, R.; Phytochemistry 1994, 37, 795 .

23. Schmidt, J.; Lien, N. T.; Khoi, N. H.; Adam, G.; Phytochemistry 1983, 22, 1032.

24. Sobrinho, D. C.; Hauptli, M. B.; Appolinário, E. V.; Kollenz, C. L. M.; Carvalho, M. G.; Braz Filho, R.; J. Braz. Chem. Soc. 1991, 2, 15.

25. Reynolds, W. F.; McLean, S.; Poplawski, J.; Enriquez, R. G.; Escobar, L. I.; I. Leon; Tetrahedron 1986, 42, 3419.

26. Aragão, P. C. A.; Toledo, J. B.; Morais, A. A.; Braz Filho, R.; Quim. Nova 1990, 13, 254.

27. Abe, F.; Chen, R. F.; Yamauchi, T.; Chem. Pharm. Bull. 1988, 36, 2784.

28. El-Naggar, L. J.; Beal, J. L.; J. Nat. Prod., 1980, 43, 649. 29. Abe, F.; Yamauchi, T.; Chem. Pharm. Bull. 1984, 32, 2947. 